\title{
A New Metal Tag for Highly Selective and Sensitive Analyses of Amino Acids and Dipeptides by HPLC/ICP-MS
}

\author{
Daigo Iwahata, Kazuki Nakamura, Rie Yamada, Hiroshi Miyano, Naoyuki Yamada
}

Institute for Innovation, Ajinomoto Co., Inc., Kawasaki, Japan.

Email: daigo_iwahata@ajinomoto.com

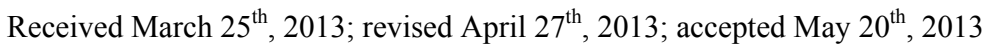

Copyright (C) 2013 Daigo Iwahata et al. This is an open access article distributed under the Creative Commons Attribution License, which permits unrestricted use, distribution, and reproduction in any medium, provided the original work is properly cited.

\begin{abstract}
We have developed a novel metal tag, bis(ethylenediamine)-4'-methyl-4-carboxybipyridine-ruthenium N-succinimidyl ester (ECRS) for sensitive analysis of amino acids using high performance liquid chromatography/inductively coupled plasma mass spectrometry (HPLC/ICP-MS). ECRS is a functional reagent, containing an ester group at one end that can be activated to bind to amino group and a chelated ruthenium at the other. The activated ester was reacted briefly with amino groups under weakly alkaline conditions. The ruthenium was detected sensitively by ICP-MS. ECRS was reacted with 17 proteinogenic amino acids in borate buffer. The derivatives were separated by reversed phase HPLC and identified by quadrupole-based ICP-MS. ECRS was suitable for speciation; low molecular weight compounds containing amino groups. We have thus established a quantitative analytical method for amino acids and dipeptides. The detection limits of branched amino acids (signal-to-noise ratio of 3) were $1.5 \mathrm{nmol} \cdot \mathrm{L}^{-1}$ in the standard solution $(100 \mathrm{amol}$ per injection).
\end{abstract}

Keywords: Metal Tag; HPLC/ICP-MS; Amino Acid; Dipeptide; Derivatization; Speciation

\section{Introduction}

Amino acids are the fundamental components of proteins and are essential for life. They are obtained from the diet and utilized throughout the body [1-3]. In addition, amino acids work as hub compounds in metabolic pathways [4], cell signaling, and other cellular phenomena. Therefore, the concentrations of amino acids in biofluids can reflect various diseases (hepatocellular carcinoma [1], malignant melanoma [5], liver disease [6,7], coronary heart disease [8], rheumatoid arthritis [9], chronic renal failure [10], diabetes mellitus [11], Alzheimer's disease $[12,13])$ and metabolic conditions.

However, the volumes of biological samples are usually very small and contain minimal amounts of free amino acids. Therefore, several highly sensitive analytical methods for amino acids have been developed [1418]. In 1958, Stein, Moore and Spackmann developed an amino acid analyzer system for the colorimetric determination of amino acids. Their method involved the production of a purple color with ninhydrin reagent [19], which was first reported by Ruhemann in 1911 [20], after stepwise separation of the amino acids on a cation exchange resin by raising the $\mathrm{pH}$ of the citric acid buffer solution. The analyzer is now capable of assaying not only protein hydrolysates but also amino acids in biological fluids, through progressive improvements to HPLC pumping performance and the development of more effective column resins. However, the sensitivity is not very high and the detection limits are a few picomoles.

In addition to the ninhydrin method, a variety of other techniques for converting amino acids to sensitively analyzable fluorescent derivatives have been developed since the late 1970s [21]. The reagents used to produce the derivatives include ortho-phthalaldehyde (OPA) [22], 9-amino quinolyl-N-hydroxysuccinimidyl carbamate (AQC) [23], and 4-fluoro-7-nitrobenzofrazane (NBD-F). [24] Their detection sensitivities are within the sub-picomole to femtomole range. Recently, mass spectrometry has also been used for this analysis, by either detecting the amino acids directly [25] or after pre-column derivatization [26,27].

The first report of the combination of immunoreaction 
and ICP-MS was described in 2001 by Zhang et al., for the determination of thyroid hormones in human serum. [28] The authors used biotinylated antibodies and $\mathrm{Eu}^{3+}$ labeled streptavidin. In 2002, the same group described the determination of thyroxin using a competitive immunoassay [29]. In the same year, Baranov et al. implemented a gel-filtration-based ICP-MS immunoassay to separate and quantify various labeled antibodies [30,31], while Zhang et al. used antibodies conjugated with colloidal gold nanoparticles for a sandwich-type immunoreaction [32]. Zhang and Baranov analyzed the metals by off-line ICP-MS after digestion.

In 2004, Whetstone et al. introduced a cysteine-specific labeling strategy, referred to as an element-coded affinity tag for peptides and proteins [33]. The reagent consists of a derivative of the bi-functional chelating agent 1,4,7,10-tetraazacyclododecane-N,N',N",N"'-tetraacetic acid (DOTA), and contains a bromoacetamido reactive group to specifically target cysteine residues. In recent approaches, two strong metal chelators (DOTA and diethylenetriamine pentaacetic acid (DTPA)) were used as metal tags with HPLC/ICP-MS [34-36]. These metal tags were suitable for size exclusion column chromatography.

In 2008, we established a highly sensitive amino acid analytical method, using reversed phase HPLC/ICP-MS. We derivatized amino acids with the commercial reagent, bis (2,2'-bipyridine)-4'-methyl-4-carboxybipyridine-ruthenium N-succinimidyl ester (BCRS). The derivatized amino acids were detected by ICP-MS. BCRS was reacted with 10 primary amino acids and the derivatives were identified [37]. However, the other proteinogenic amino acids were not separated. The BCRS derivatives, which included two bipyridines in the compounds, were too bulky to separate by reversed phase HPLC. This reagent was not enough for speciation. In this study, we have designed and synthesized a new metal tag, bis (ethylenediamine)-4'-methyl-4-carboxybipyridine-ruthenium $\mathrm{N}$-succinimidyl ester (ECRS). ECRS was reacted with 17 proteinogenic amino acids (except for unstable amino acids in the stock and the buffer solutions; glutamine, asparagine and tryptophan) and 3 common dipeptides, in the presence of borate buffer. It was suitable for speciation.

\section{Experimental}

\subsection{Chemicals and Reagents}

Reagent grade acetonitrile, acetic acid, ammonium hydroxide, ammonium acetate and sodium borate were purchased from Wako Pure Chemical Industries, Ltd. (Osaka, Japan). 4'-Methyl-2,2'-bipyridine-4-carboxylic acid was obtained from Rubypy Scientific Inc. (Ottawa, Canada). Bis(2,2-bipyridine)-4-methyl-4-carboxybipyri- dine-ruthenium N-succinimidyl ester-bis(hexafluorophosphate) (=BCRS), all amino acids, diamines, and other reagents for synthesis were obtained from Fluka Chemie AG/Sigma-Aldrich (St Louis, MO, USA). Distilled deionized water (18.3 MO) was obtained from a Millipore water purifying system (Bedford, MA, USA).

\subsection{Design of a New Metal Tag}

The image of the metal tag for low molecular compounds is shown in Figure 1. This metal tag contains the reactive group that can be activated to react with amino acids and the chelated metal. The nature of the chelating group is hydrophobic. The hydrophobicity of the chelation moiety is very important. The suitable hydrophobicity is needed to retain and separate the low molecular compounds in the reverse phase column.

\subsection{Synthesis of the New Metal Tag}

The synthesis scheme for the new metal tag reagent is shown in Figure 2, and has been previously described [38]. Ruthenium chloride $(1.0 \mathrm{~g}=3.82 \mathrm{mmol})$ was dissolved in water/ethanol $(\mathrm{v} / \mathrm{v}=16 \mathrm{~mL} / 4 \mathrm{~mL})$, and the solution was heated to $50^{\circ} \mathrm{C}$. 4'-Methyl-2,2'-bipyridine-4carboxylic acid $(778 \mathrm{mg}=3.6 \mathrm{mmol})$ was dissolved in 20 $\mathrm{mL}$ DMF and dripped into the ruthenium solution slowly, under an argon atmosphere. The mixture was heated to $80^{\circ} \mathrm{C}$ and stirred for $4 \mathrm{~h}$. It was cooled to room temperature and stored at $4{ }^{\circ} \mathrm{C}$ overnight. On the following day, it was warmed to room temperature, and ethylene diamine $(2.55 \mathrm{~mL})$ was added. The reaction solution was heated to $80^{\circ} \mathrm{C}$ and stirred for $6 \mathrm{~h}$, then concentrated under reduced pressure, and boiled with $10 \mathrm{~mL}$ isopropanol. The residue was combined with $50 \mathrm{~mL}$ pure water and $8 \mathrm{~mL}$ $60 \%$ hexafluorophosphoric acid, followed by $20 \mathrm{~mL}$ saturated hexafluorophosphoric acid. The separated crystals were washed with $20 \mathrm{~mL}$ pure water and dried under reduced pressure (Compound 1, $2.89 \mathrm{mmol}$, yield: 76\%). Compound $1(2.0 \mathrm{~g}=2.75 \mathrm{mmol})$ was dissolved in 40

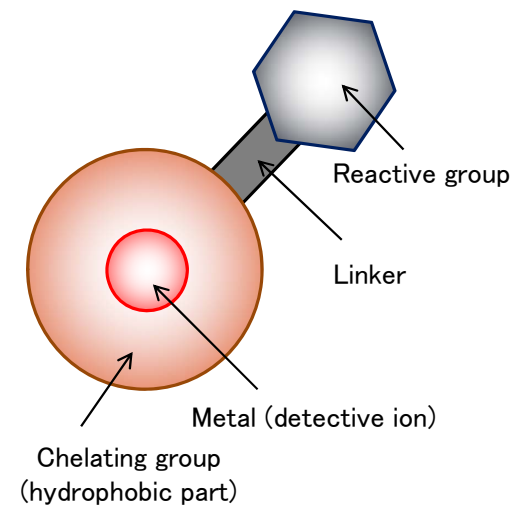

Figure 1. Constitution of the metal-coded affinity tag for low molecular compounds (schematic view). 


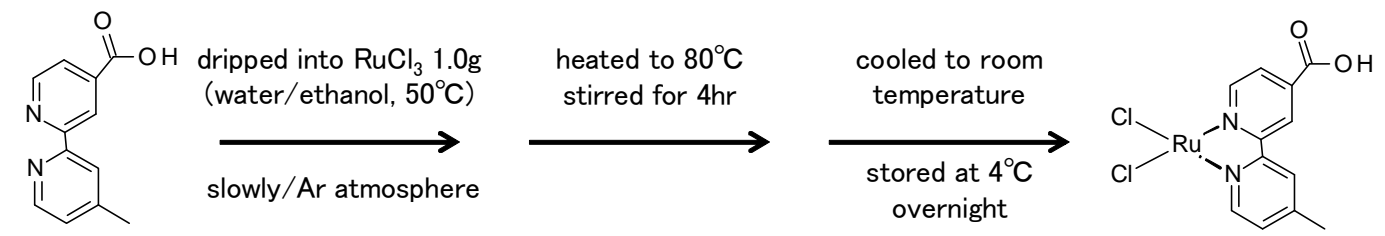

$778 \mathrm{mg}$ (in DMF)
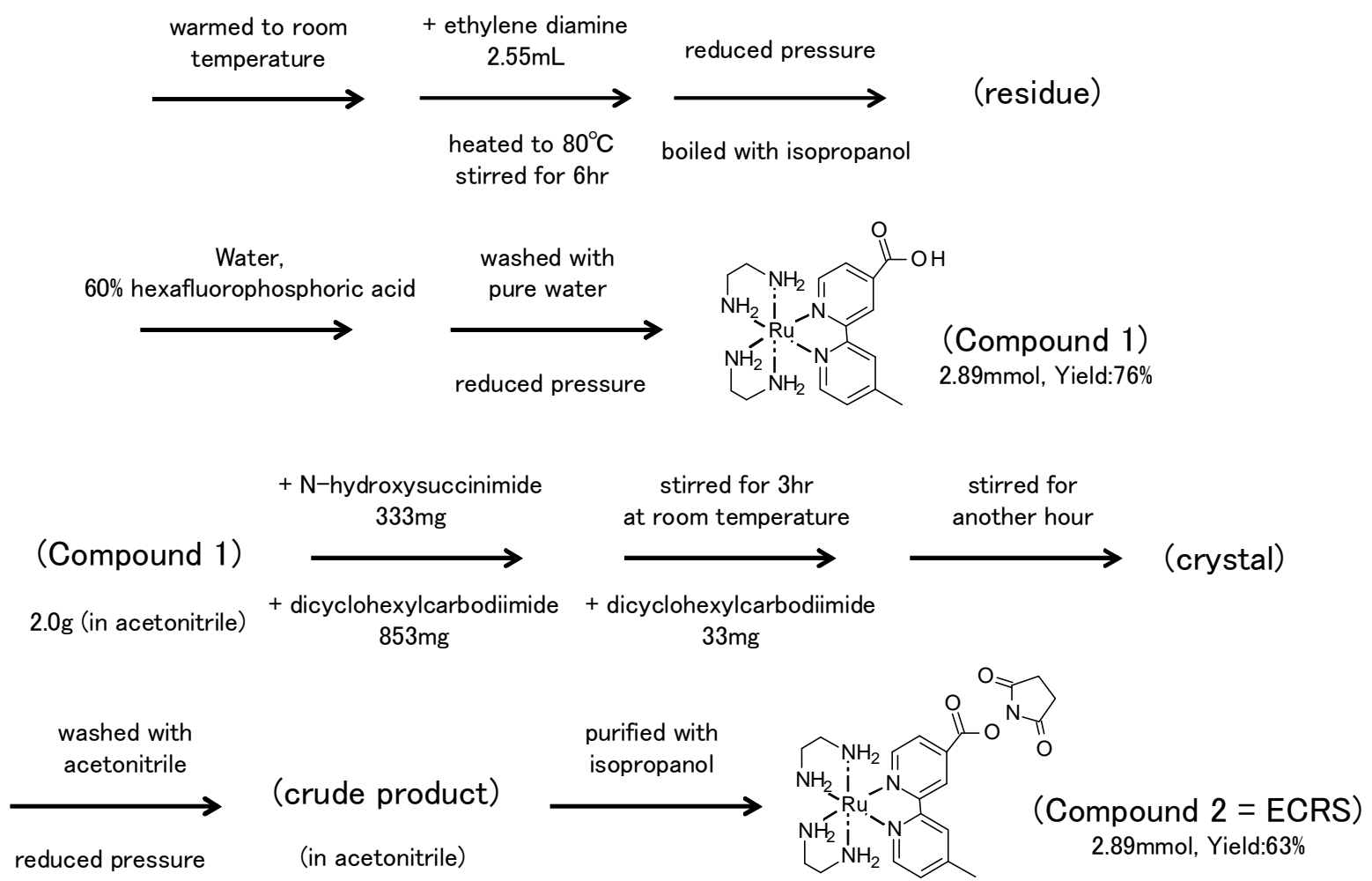

Figure 2. Synthesis scheme of the metal-coded affinity tag.

$\mathrm{mL}$ acetonitrile, then $333 \mathrm{mg} \mathrm{N}$-hydroxysuccinimide and $853 \mathrm{mg}$ dicyclohexylcarbodiimide were added. The solution was stirred at room temperature for $3 \mathrm{~h}$, and after dicyclohexylcarbodiimide (33 mg) was added, it was stirred for another hour. The remaining crystals were washed with acetonitrile and concentrated under reduced pressure. The crude product was dissolved in $5 \mathrm{~mL}$ acetonitrile and purified with isopropanol (Compound $2=$ ECRS, 2.89 mmol, yield 63\%).

\subsection{Instrumentation}

HPLC experiments were performed with a Shimadzu modular system equipped with a control unit (CBM-20A), autosampler (SIL-20AC HT UFLC), UV detector (SPD20A UFLC), column oven (CTO-20AC), two pumps (LC-20ADXR UFLC) and degasser (DGU-20A3). The system was coupled to a quadrupole-based ICP-MS (7700, Agilent Co., Inc., USA). An LC/MS system was used for Q-TOF MS (Q-TOF Premier, Waters Corporation, MA, USA).

\subsection{ICP-MS Parameters}

The sampling and skimmer cones were both composed of nickel. The rates of plasma gas flow, auxiliary gas flow and carrier gas flow were 15.0, 1.0 and $1.0-1.2 \mathrm{~L} \cdot \mathrm{min}^{-1}$, respectively. The collision gas $(\mathrm{He})$ flow rate was 4.3 $\mathrm{mL} \cdot \mathrm{min}^{-1}$. High temperature He mode was effective in decreasing the back ground value. The radio frequency (RF) power was maintained at $1590 \mathrm{~W}$. The sampling depth was set between 6 and $7 \mathrm{~mm}$. The ICP-MS parameters were optimized for each investigation to achieve the best signal to noise ratio, using a standard solution containing $1 \mathrm{ppb}$ each of lithium, yttrium and thallium. The sensitivity of the yttrium ion was about $150,000 \mathrm{cps} / \mathrm{ppb}$. Detection was performed using ICP$\mathrm{MS}$, with ruthenium ion-selective detection at $\mathrm{m} / \mathrm{z}=101$ or 102 .

\subsection{HPLC Conditions}

The derivatized amino acids were injected into an Inertsil ODS-3 column, $3 \mu \mathrm{m}$ particle size, $1.0 \mathrm{~mm}$ i.d. $\times 150$ 
mm (GL Science Instruments, Inc. Tokyo, Japan). Mobile phase A was $10 \mathrm{mmol} \cdot \mathrm{L}^{-1}$ acetate buffer ( $\left.\mathrm{pH} 4.8\right)$. The acetate buffer was prepared as follows: $120 \mu \mathrm{L}$ of acetic acid and $3.7 \mathrm{~g}$ of ammonium acetate were mixed and dissolved in $1 \mathrm{~L}$ of distilled, deionized water. Mobile phase $\mathrm{B}$ was acetonitrile-water $(\mathrm{v} / \mathrm{v}=60: 40)$. Each mobile phase was filtered through a $0.45 \mu \mathrm{m}$ membrane (Nalge Nunc International, Rochester, NY, USA).

The flow rate was $50 \mu \mathrm{L} \cdot \mathrm{min}^{-1}$, and the injection volume was $1 \mu \mathrm{L}$. The following gradient program was used: 0 - $5 \min 5 \%, 5$ - 40 min to $35 \%, 40$ - $55 \mathrm{~min} 100 \%$ and 55 - 70 min $5 \%$ mobile phase B. The column was run at room temperature (about $25^{\circ} \mathrm{C}$ ). The sample tray temperature was $4^{\circ} \mathrm{C}$.

\subsection{Q-TOFMS Parameters}

Mass spectrometry was performed in the ESI positive mode. The desolvation gas was set to $700 \mathrm{~L} \cdot \mathrm{h}^{-1}$ at a desolvation temperature of $120^{\circ} \mathrm{C}$, the cone gas was set to $50 \mathrm{~L} \cdot \mathrm{h}^{-1}$, and the source temperature was set to $80^{\circ} \mathrm{C}$. The capillary and cone voltages were set to 3000 and 20 $\mathrm{V}$, respectively. The MCP detector voltage was set to 1 , $600 \mathrm{~V}$. Argon was employed as the collision gas. The collision gas flow was $0.6 \mathrm{~mL} \cdot \mathrm{min}^{-1}$ and the collision energy was $4 \mathrm{~V}$. The scan range was from 100 to 1000 $\mathrm{m} / \mathrm{z}$.

\subsection{Amino Acid and Dipeptide Derivatization with the New Metal Tag (ECRS)}

The derivatization of glutamic acid with ECRS is shown in Figure 3. ECRS was prepared by dissolution to a concentration of $10 \mu \mathrm{mol} \cdot \mathrm{L}^{-1}$ in acetonitrile, just before use. It was stored in a case at room temperature. Stock solutions of $0.1 \mu \mathrm{mol} \cdot \mathrm{L}^{-1}$ of each amino acid and dipeptide, dissolved in $0.1 \mathrm{~mol} \cdot \mathrm{L}^{-1} \mathrm{HCl}$, were prepared. They were properly diluted and mixed before use. A $10 \mu \mathrm{L}$ aliquot of the sample solution was mixed with $60 \mu \mathrm{L}$ of $0.2 \mathrm{~mol} \cdot \mathrm{L}^{-1}$ sodium tetraborate $(\mathrm{pH} 8.0)$ and $20 \mu \mathrm{L}$ of acetonitrile, and then a $10 \mu \mathrm{L}$ portion of the tag solution was added. The metal tag was present in excess, relative to the amino acids and dipeptides in the reaction mixture. After mixing for $1 \mathrm{~min}$ using a vortex mixer, the mixture was heated at $55^{\circ} \mathrm{C}$ for $5 \mathrm{~min}$ in an aluminum bath. A 50 $\mu \mathrm{L}$ aliquot of $1 \mathrm{~mol} \cdot \mathrm{L}^{-1}$ ammonium acetate buffer $(\mathrm{pH}$
4.8) was added to the reaction mixture, which was then thoroughly mixed with a vortex mixer. The mixture was stored in a tightly closed container at $4^{\circ} \mathrm{C}$ until it was subjected to HPLC/ICP-MS.

\subsection{Analyses of Amino Acid and Dipeptide Derivatives}

The amino acid and dipeptide derivatives were detected by HPLC, HPLC/ICP-MS, and LC-Q-TOFMS. Each amino acid and dipeptide derivatized with ECRS was detected at $254 \mathrm{~nm}$, using the UV detector. Mixed amino acids and dipeptides derivatized together were discovered at $\mathrm{m} / \mathrm{z}=101$, using HPLC/ICP-MS. To combine HPLC with ICP-MS, the HPLC outlet $\left(0.05 \mathrm{~mL} \cdot \mathrm{min}^{-1}\right)$ was connected with PEEK tubing via a ceramic valve to a micro concentric nebulizer within a spray chamber. The mass spectra of the derivatives were obtained using LCQ-TOFMS.

\section{Results}

We designed and synthesized a new metal tag (ECRS). ECRS is a functional reagent with reactivity to amine group and it contains the chelating group, too. The chelate of ECRS with ruthenium had suitable hydrophobicity and size for analysis by HPLC, using a C-18 reversed phase column. It was smaller than the strong hydrophobic tag (BCRS) used in our previous study.

In total, $1.4 \mathrm{~g}$ (yield $63 \%$ ) of ECRS was obtained using our synthesis scheme. The ECRS was kept at room temperature until use. Its activity remained constant over a year.

We derivatized branched amino acids (BCAAs) with ECRS at various incubation times (5, 30, 60, 90, 120 min). The amino acids and dipeptides rapidly derivatized within 5 min with ECRS. In contrast, the time needed to react amino acids with BCRS was $90 \mathrm{~min}$.

The chromatograms of 17 primary and secondary amino acids and 3 dipeptides derivatized with ECRS are shown in Figure 4. Each amino acid and dipeptide sample was prepared independently. We have recorded the UV chromatogram at $254 \mathrm{~nm}$ for each sample. The excess unreacted metal tag eluted before many amino compound derivatives, except for the acidic amino acids (glutamic acid and aspartic acid), formed. Lysine, which has two

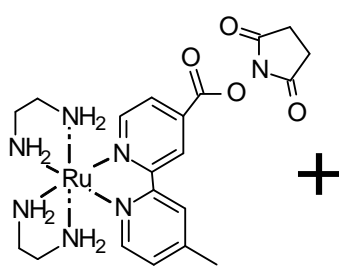<smiles>NC(CCC(=O)O)C(=O)O</smiles>
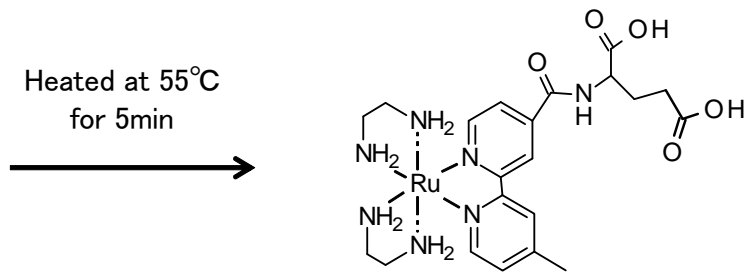

Figure 3. Derivatization of glutamic acid with the ECRS. 


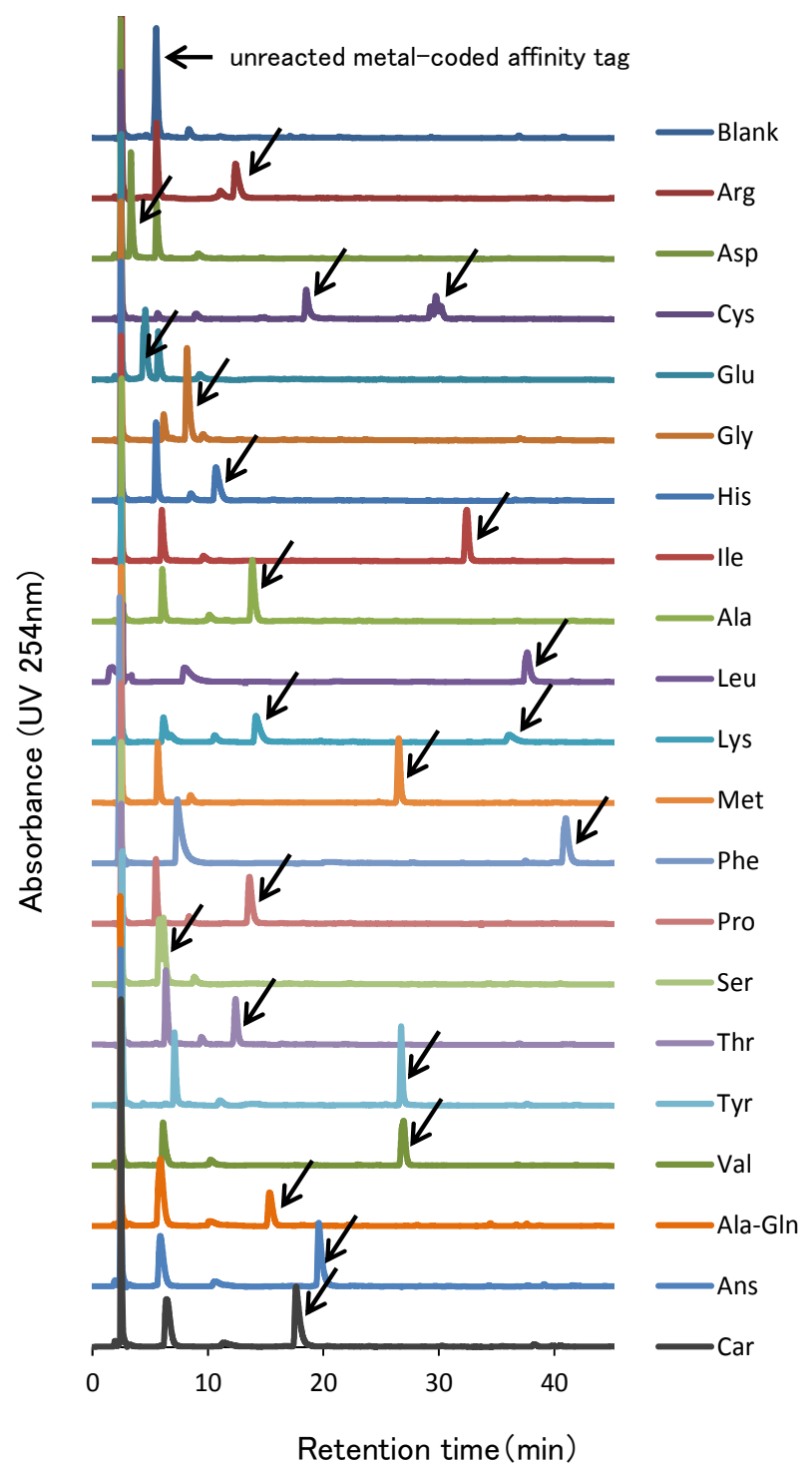

Figure 4. The Chromatograms of amino acids and dipeptides.

amino groups in the compound, formed two types of derivatives. We acquired the mass spectra of the derivatives by LC-Q-TOFMS, as shown in Figure 5.

The mixture of amino acids and dipeptides was derivatized with ECRS. The chromatogram for this solution, detected by HPLC/ICP-MS $(\mathrm{m} / \mathrm{z}=101)$, is shown in Figure 6. We used to detect $\mathrm{m} / \mathrm{z}=101$ or 102 . The signal intensity at $\mathrm{m} / \mathrm{z}=102$ increased than $\mathrm{m} / \mathrm{z}=101$, but background value was higher, too. We confirmed that the amino acids and the dipeptides were simultaneously derivatized.

The derivatization with ECRS was suitable for the quantitative analysis of amino acids. The calibration curves of BCAAs, in the concentration range of 0.75 $750 \mu \mathrm{mol} \cdot \mathrm{L}^{-1}$ in the standard solution $(0.05-50 \mathrm{pmol}$ per injection), are shown in Figure 7.
The detection limit of BCAAs was $1.5 \mathrm{nmol} \cdot \mathrm{L}^{-1}$ in the standard solution (100 amol per injection) (signal to noise ratio $=3$ ). The peak shapes of the dipeptides and threonine were broader than those of the BCAAs. The detection limit of the dipeptides and threonine was 15 $\mathrm{nmol} \cdot \mathrm{L}^{-1}$ under the standard solution conditions ( $1 \mathrm{fmol}$ per injection). The correlation coefficients of the derivatized BCAAs were $\mathrm{r}^{2}=0.999$. The relative standard deviation of $1.5 \mu \mathrm{mol} \cdot \mathrm{L}^{-1}$ of the BCAA adducts was $1.5 \%$ $(n=5)$.

\section{Discussion}

To develop a new metal tag for HPLC/ICP-MS, we had to maintain the bond between the amino acid and the metal moiety in an aqueous solution, such as the HPLC mobile phase, and to separate the amino acid derivatives by reversed phase HPLC.

Most metal tags are a bi-functional reagent with a group that can be activated towards amino acids and a metal-binding group. Metal binding can, in general, be achieved with a chelator. Our novel metal tag was also the chelate compound, because we chose ethylenediamine as the metal chelator. The ruthenium ion remained associated with the complex under our HPLC conditions, because the ion was not detected in the void volume. We tried to synthesize the metal tag with the different metals (ex. indium and rare earth elements), but they were not stable.

The separation ability by HPLC was also important. The first major metal tag employed was the DOTA cys-specific labeling technique. It was recently reported that DTPA reacted with the amino terminus and the internal Lys. Both of these methods derivatized proteins and peptides. They are not suitable to analyze low molecular weight compounds, such as amino acids and dipeptides. It is too hydrophilic to separate with reverse phase column. On the other hand, reversed phase HPLC methods for the quantification and separation of amino acids were developed and we subsequently designed our new metal tag. Our tag was more hydrophobic than those previously used for proteins. Furthermore, it was superior because the metal chelator was not too large. The ethylenediamine moiety was more favorable for separation, compared with the bipyridine moiety.

We confirmed the structures of the derivatives by LCQ-TOFMS (scan range 100 to $1000 \mathrm{~m} / \mathrm{z}$ ). We detected the precursor ion of the amino acid and dipeptide derivatives (Figure 5). The double charged ion was stronger than the single charged ion (Figures 5(a), (c) and (e)). The chemical formula and formula weight of delivertized amino acids and dipeptide are shown in Table 1. All single charged precursor ions of the derivatives were in good agreement with the exact masses (Figures 5(b), 

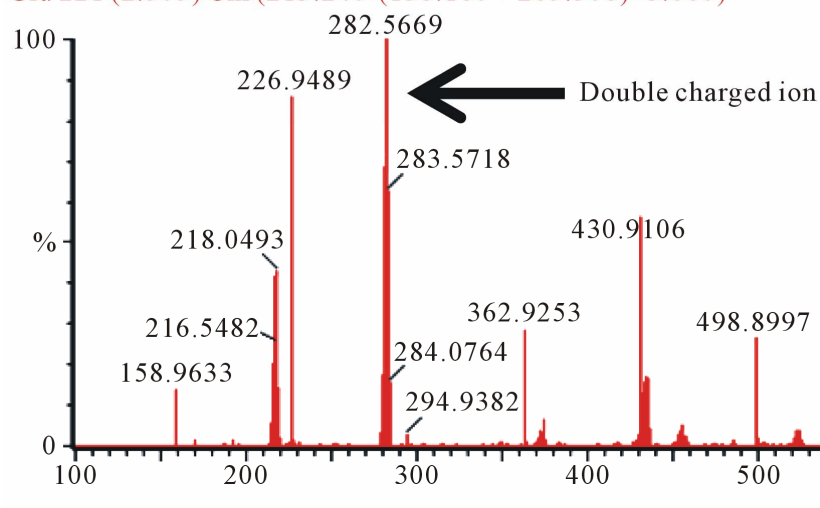

1: TOF MS ES +

$1: \mathrm{TOF}$ MS ES+
$1.88 \mathrm{e} 4$

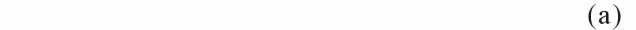

Glu $221(2.505)$ Cm $(213: 240-(138: 180+265: 308) \times 3.000)$

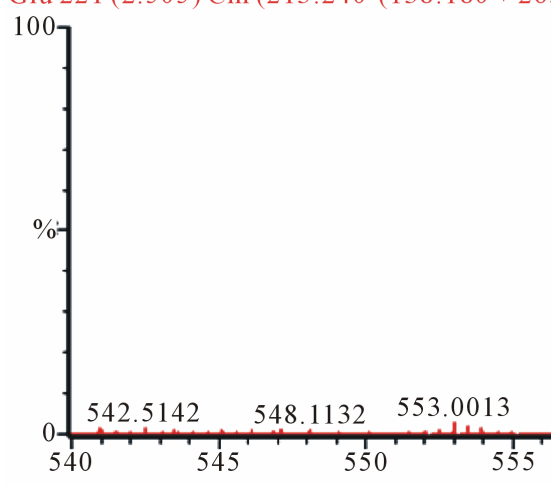

(a)
566.8860

85

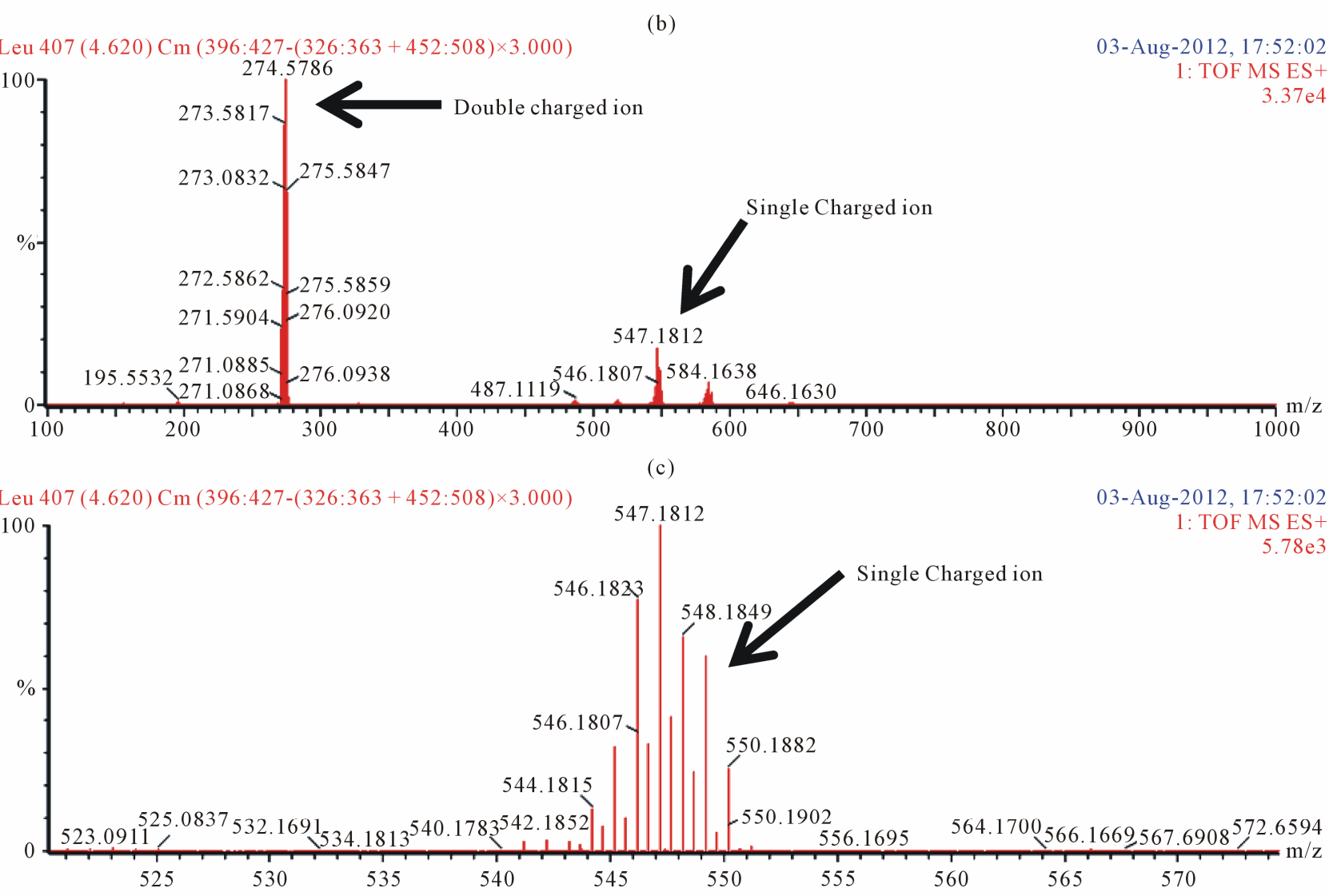

(d) 


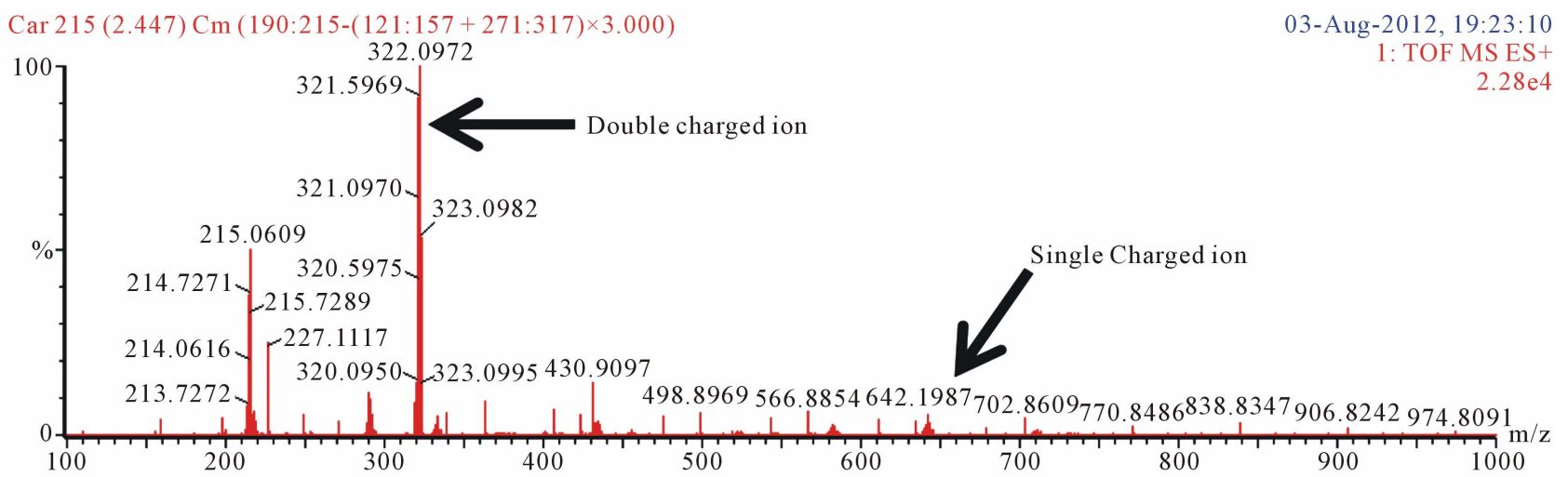

(e)

Car 215 (2.447) Cm (190:215-(121:157+271:317) ×3.000)

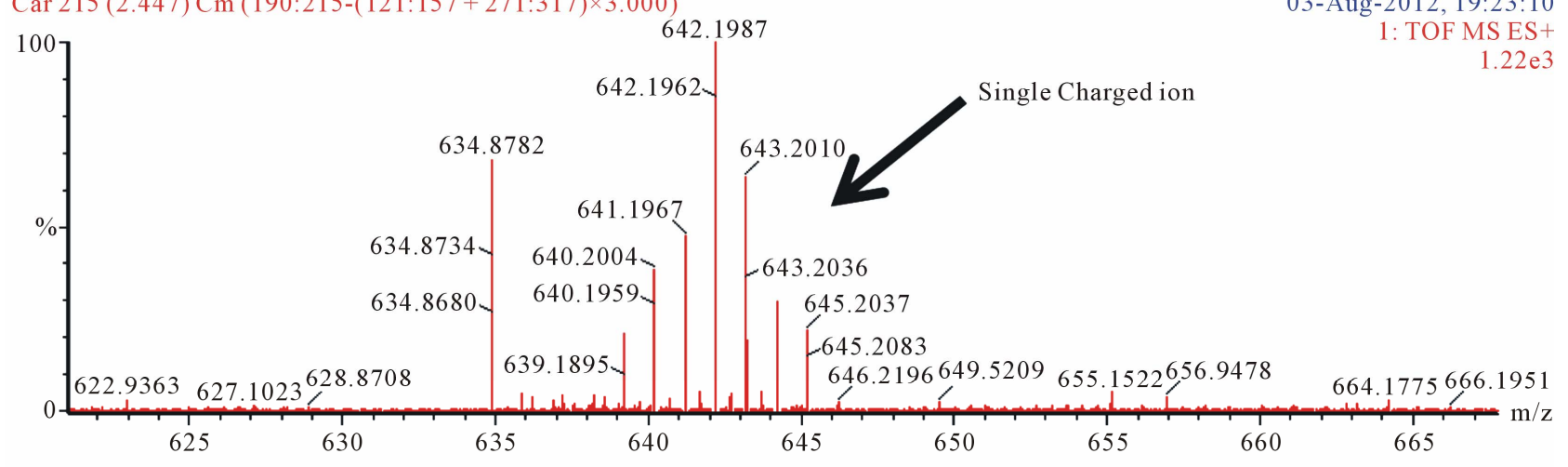

(f)

Figure 5. The mass spectra of delivertized amino acids and dipeptide. (a) Glutamic acid; scan range from 100 to $1000 \mathrm{~m} / \mathrm{z}$; (b) Glutamic acid; scan range from 540 to $595 \mathrm{~m} / \mathrm{z}$; (c) Leucine; scan range from 100 to $1000 \mathrm{~m} / \mathrm{z}$; (d) Leucine; scan range from 520 to $575 \mathrm{~m} / \mathrm{z}$; (e) Carnosine; scan range from 100 to $1000 \mathrm{~m} / \mathrm{z}$; (f) Carnosine; scan range from 620 to $570 \mathrm{~m} / \mathrm{z}$,

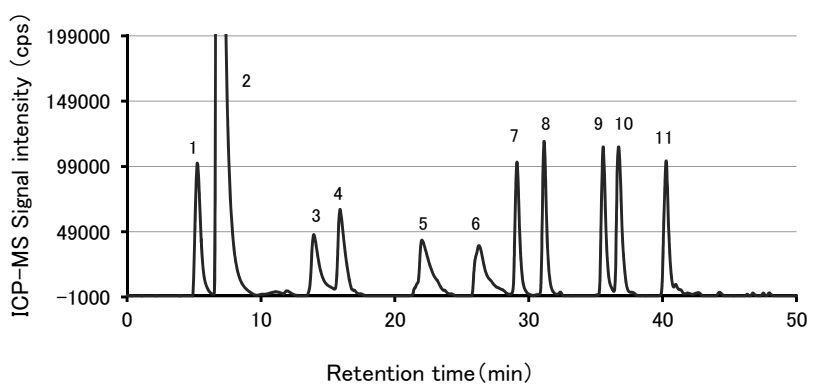

Figure 6. The chromatogram of 8 amino acids and 2 dipeptides derivatized with $\mathrm{ECRS}(\mathrm{m} / \mathrm{z}=101)$. The concentration of all amino compounds in the stock solution is $75 \mu \mathrm{mol} \cdot \mathrm{L}^{-1}$. 1. glutamic acid, 2. unreacted ECRS, 3. threonine, 4. Lalanine, 5. carnosine, 6 . anserine, 7 . methionine 8. valine, 9, isoleucine, 10. leucine, 11. phenylalanine.

Table 1. The chemical formula and formula weight of delivertized amino acid and dipeptide.

\begin{tabular}{ccc}
\hline & Chemical Formula & Formula Weight \\
\hline Glu & $\mathrm{C}_{22} \mathrm{H}_{34} \mathrm{~N}_{6} \mathrm{O}_{5} \mathrm{Ru}$ & 563.621 \\
Leu & $\mathrm{C}_{23} \mathrm{H}_{38} \mathrm{~N}_{6} \mathrm{O}_{3} \mathrm{Ru}$ & 547.666 \\
Car & $\mathrm{C}_{27} \mathrm{H}_{40} \mathrm{~N}_{8} \mathrm{O}_{4} \mathrm{Ru}$ & 641.739 \\
\hline
\end{tabular}

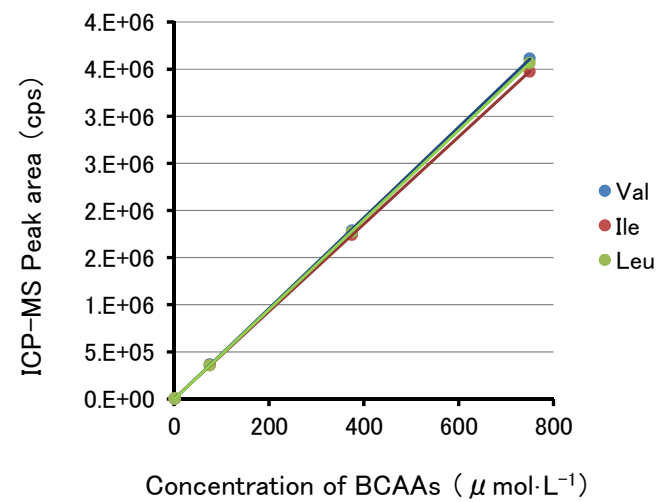

Figure 7. The calibration curves of the BCAAs. The vertical axis was the peak area with ICP-MS. The horizontal axis is concentration of BCAAs in the analytical solution. The correlation coefficients are $r_{2}=0.999\left(\right.$ from $0.75-750 \mu \mathrm{mol} \cdot \mathrm{L}^{-1}$ ). The relative standard deviations through all analytical process are $1.5 \%\left(\right.$ at $\left.1.5 \mu \mathrm{mol} \cdot \mathrm{L}^{-1}, \mathrm{n}=5\right)$.

(d) and (f)). It was identified that the amino acids and dipeptides were deivatized as expected.

We separately derivatized each amino acid and dipeptide, and monitored them with a HPLC/UV detector (Figure 4). All derivatives were separated from the un- 
reacted metal tag. We could detect 17 proteinogenic amino acids. The secondary amino acid, proline, was also derivatized. Proline was not derivatized by previous metal tag, BCRS. BCRS only derivatized primary amino acids. The lysine and cysteine derivatives generated two peaks, because they had two reaction sites. Although we derivatized lysine and cysteine at some incubation times, two kinds of derivatives, bound with one or two ECRS moieties, were observed. In this study, one mole of lysine did not react completely with two moles of the metal tag at the same condition of the other amino acids. To achieve the optimal derivatization condition for lysine and cysteine, the concentration of the metal tag, the $\mathrm{pH}$ of the borate buffer and the temperature of reaction should be individually explored.

The reaction time for the derivatization with ECRS was very short. The process was completed within $5 \mathrm{~min}$. In a previous report, the derivatization reaction with BCRS took $1.5 \mathrm{~h}$. The small chelator probably had a positive influence on reducing the reaction rate. In addition, we only considered the reaction time, but whereas ECRS can be left at room temperature, BCRS, in contrast, must be kept at $-20^{\circ} \mathrm{C}$.

In our analytical method, the amino acids and dipeptides were combined with the ruthenium atom by ECRS, and were detected with same sensitivity as the ruthenium ion. The actual detection limits of the amino acids through all processes were about 100 amol, which is better than the fluorescent analytical method [14,15], equivalent to the LC/MS/MS method (after pre-column derivatization) $[17,18]$ and our previous method $[37,39]$. The calibration curves for the BCAAs are shown in Figure 7. The three curves exhibit equal slopes and intensities. In Figure 4, the peak heights of the derivatives differed but the peak areas were the same. The detection limits of dipeptides and threonine were higher than those of the other amino acids, because these peaks were broadened under our HPLC conditions for the comprehensive analysis. The sensitivity might be improved by optimizing the gradient elution method for each compound.

\section{Conclusion}

The new analytical method using the metal tag with HPLC/ICP-MS is not significantly affected by the sample matrix. ICP is one of the most powerful ion sources and it has high temperature (between 6000 and 10,000 K) and high electron number density (between $6.4 \times 10^{14}$ and $1.2 \times 10^{15} \mathrm{~cm}^{-1}$ ). All analytes and the sample matrix are ionized at once. On the other hand, LC-MS is powerful tool that is very sensitive and selective, but it is often affected by impurities. When the analyte and the impurity elute at the same time, the intensity of the ana- lyte is decreased, obviously. In some cases, we cannot obtain analytical values. In our method, we detected the ruthenium ion in the metal tag. This will be useful, because all analytes are detected with high accuracy and the same sensitivity regardless of sample matrix. In this study, we have designed and synthesized a new ruthenium complex, and have shown that our method could be developed for speciation further. For example, by changing the reactive functional group of the metal tag, it could be applied to another low molecular weight compounds, such as carboxylic acids.

\section{Acknowledgements}

The authors thank the synthesis team at the Nard Institute, Ltd., for support in the synthesis of the new metal tag compound.

We are grateful to Ms. Sachise Karakawa for providing the MS spectra from LC/MS measurements.

\section{REFERENCES}

[1] A. Watanabe, T. Higashi, T. Sakata and H. Nagashima, "Serum Amino Acid Levels in Patients with Hepatocellular Carcinoma," Cancer, Vol. 54, No. 9, 1984, pp. 1875-1882.

doi:10.1002/1097-0142(19841101)54:9<1875::AID-CNC $\underline{\mathrm{R} 2820540918>3.0 . \mathrm{CO} ; 2-\mathrm{O}}$

[2] K. J. Carpenter, A. E. Harper and R. E. Olson, "Experiments that Changed Nutritional Thinking," The Journal of Nutrition, Vol. 127, No. 5, 1997, pp. 1017S-1053S.

[3] R. M. Jennie, "Protein and Amino Acid Metabolism during and after Exercise and the Effects of Nutrition," Annual Review of Nutrition, Vol. 20, 2000, pp. 457-483. doi:10.1146/annurev.nutr.20.1.457

[4] A. Wagner and D. Fell, "The Small World inside Large Metabolic Networks," Proceedings of Royal Society, Vol. 268, No. 1478, 2001, pp. 1803-1810. doi:10.1098/rspb.2001.1711

[5] G. Weinlich, C. Murr, L. Richardsen, C. Winkler and D. Fuchs, "Decreased Serum Tryptophan Concentration Predicts Poor Prognosis in Malignant Melanoma Patients," Dermatology, Vol. 214, 2007, 214, pp. 8-14. doi:10.1159/000096906

[6] E. Holm, O. Sedlaczek and E. Grips, "Amino Acid Metabolism in Liver Disease," Current Opinion in Clinical Nutrition \& Metabolic Care, Vol. 2, No. 1, 1999, pp. 4753. doi:10.1097/00075197-199901000-00009

[7] M. Holecek, "Three Targets of Branched-Chain Amino Acid Supplementation in the Treatment of Liver Disease," Nutrition, Vol. 26, No. 5, 2010, pp. 482-490. doi:10.1016/j.nut.2009.06.027

[8] O. A. Obeid, "Plasma Amino Acid Concentrations in Patients with Coronary Heart Disease: A Comparison between U.K. Indian Asian and Caucasian Men," International Journal for Vitamin and Nutrition Research, Vol. 75, No. 4, 2005, pp. 267-273. 
doi:10.1024/0300-9831.75.4.267

[9] D. Gerber, "Decreased Concentration of Free Histidine in Serum in Rheumatoid Arthritis, an Isolated Amino Acid Abnormality Not Associated with Generalized Hypoaminoacidemia," The Journal of Rheumatology, Vol. 2, 1975, pp. 384-392.

[10] Q. Zhang, M. Takahashi, Y. Noguchi, T. Sugimoto, et al., "Plasma Amino Acid Profiles Applied for Diagnosis of Advanced Liver Fibrosis in Patients with Chronic Hepatitis C Infection," Hepatology Research, Vol. 34, 2006, pp. 170-177. doi:10.1016/j.hepres.2005.12.006

[11] Y. Ohtsuka and Y. Agishi, "Abnormal Amino Acid Metabolism in Diabetes Mellitus," Nippon Rinsho, Vol. 50, 1992, pp. 1631-1636.

[12] A. Mochizuki, A. Tamaoka, A. Shimohata, Y. Komatsuzaki and S. I. Shoji, "Ab42-Positive Non-Pyramidal Neurons around Amyloid Plaques in Alzheimer's Disease," Lancet, Vol. 355, No. 9197, 2000, pp. 42-44. doi:10.1016/S0140-6736(99)04937-5

[13] A. R. Hipkis, "Carnosine, Diabetes and Alzheimer's Desease," Expert Review of Neurotherapeutics, Vol. 9, No. 5, 2009, pp. 583-585. doi:10.1586/ern.09.32

[14] T. Santa, A. Takeda, S. Uchiyama, T. Fukushima, et al., "N-(4-Nitro-2,1,3-benzoxadiazoyl-7-yl)-N-methyl-2-amin oacetohydrazide (NBD-CO-Hz) as a Precolumn Fluorescent Derivatization Reagent for Carboxylic Acids in High-Performance Liquid Chromatography," Journal of Pharmaceutical and Biomedical Analysis, Vol. 17, No. 6-7, 1998, pp. 1065-1070. doi:10.1016/S0731-7085(98)00072-7

[15] S. Uchiyama, T. Santa, N. Okiyama, T. Fukushima and K. Imai, "Fluorogenic and Fluorescent Labeling Reagents with a Benzofurazan Skeleton," Biomedical Chromatography, Vol. 15, No. 5, 2001, pp. 295-318. doi:10.1002/bmc. 75

[16] S. Iwatani, S. V. Dien, K. Shimbo, K. Kubota, et al., "Determination of Metabolic Flux Changes during Fed-Batch Cultivation from Measurements of Intracellular Amino Acids by LC-MS/MS," Journal of Biotechnology, Vol. 128, No. 1, 2007, pp. 93-111. doi:10.1016/j.jbiotec.2006.09.004

[17] K. Shimbo, A. Yahashi, K. Hirayama, M. Nakazawa and H. Miyano, "Multifunctional and Highly Sensitive Precolumn Reagents for Amino Acids in Liquid Chromatography/Tandem Mass Spectrometry," Analytical Chemistry, Vol. 81, No. 13, 2009, pp. 5172-5179. doi:10.1021/ac900470w

[18] K. Shimbo, T. Oonuki, A. Yahashi, K. Hirayama and H. Miyano, "Precolumn Derivatization Reagents for HighSpeed Analysis of Amines and Amino Acids in Biological Fluid Using Liquid Chromatography/Electrospray Ionization Tandem Mass Spectrometry," Rapid Communications in Mass Spectrometry, Vol. 23, No. 10, 2009, pp. 1483-1492. doi:10.1002/rcm.4026

[19] D. H. Spackman, W. H. Stein and S. Moore, "Automatic Recording Apparatus for Use in the Chromatography of Amino Acids," Analytical Chemistry, Vol. 30, No. 7, 1958, pp. 1190-1206. doi:10.1021/ac60139a006

[20] S. Ruhemann, "Triketohydrindene Hydrate. Part IV. Hy- drindantin and Its Analogues," Journal of the Chemical Society, Vol. 99, 1911, pp. 1306-1310. doi:10.1039/ct9119901306

[21] S. A. Cohen and D. J. Strydom, "Amino Acid Analysis Utilizing Phenylisothiocyanate Derivatives," Analytical Biochemistry, Vol. 174, No. 1, 1988, pp. 1-16. doi:10.1016/0003-2697(88)90512-X

[22] B. S. Glaeser and T. A. Hare, "Measurement of GABA in Human Cerebrospinal Fluid," Biochemical Medicine, Vol. 12, 1975, pp. 274-282. doi:10.1016/0006-2944(75)90129-5

[23] S. A. Cohen and K. M. D. Antonis, "Applications of Amino Acid Derivatization with 6-Aminoquinolyl-N-hydroxysuccinimidyl Carbamate Analysis of Feed Grains, Intravenous Solutions and Glycoproteins," Journal of Chromatography A, Vol. 661, No. 1-2, 1994, pp. 25-34. doi:10.1016/0021-9673(93)E0821-B

[24] Y. Watanabe and K. Imai, "Serum Amino Acid Levels in Patients with Hepatocellular Carcinoma," Analytical Biochemistry, Vol. 116, No. 2, 1981, pp. 471-472. doi:10.1016/0003-2697(81)90390-0

[25] M. Piraud, C. Vianey-Saban, K. Petritis, C. Elfakir, et al., "ESI-MS/MS Analysis of Underivatised Amino Acids: A New Tool for the Diagnosis of Inherited Disorders of Amino Acid Metabolism. Fragmentation sTudy of 79 Molecules of Biological Interest in Positive and Negative Ionisation Mode," Rapid Communications in Mass Spectrometry, Vol. 17, No. 12, 2003, pp. 1297-1311. doi: $10.1002 / \mathrm{rcm} .1054$

[26] W. C. Yang, H. Mirzaei, X. Liu and F. Regnier, "Enhancement of Amino Acid Detection and Quantification by Electrospray Ionization Mass Spectrometry," Analytical Chemistry, Vol. 78, No. 13, 2006, pp. 4702-4708. doi:10.1021/ac0600510

[27] P. Ross, Y. Huang, J. Marchese, B. Williamson, et al., "Multiplexed Protein Quantitation in Saccharomyces Cerevisiae Using Amine-Reactive Isobaric Tagging Reagents," Molecular \& Cellular Proteomics, Vol. 3, 2004, pp. 1154-1169. doi:10.1074/mcp.M400129-MCP200

[28] C. Zhang, F. Wu, Y. Zhang, X. Wang and X. Zhang, “A Novel Combination of Immunoreaction and ICP-MS as a Hyphenated Technique for the Determination of Thyroid-Stimulating Hormone (TSH) in Human Serum," Journal of Analytical Atomic Spectrometry, Vol. 16, No. 12, 2001, pp. 1393-1396. doi:10.1039/b106387c

[29] C. Zhang, F. Wu and X. Zhang, "ICP-MS-Based Competitive Immunoassay for the Determination of Total Thyroxin in Human Serum," Journal of Analytical Atomic Spectrometry, Vol. 17, No. 10, 2002, pp. 13041307. doi: $10.1039 / \mathrm{b} 205623 \mathrm{~b}$

[30] Z. A. Quinn, V. I. Baranov, S. D. Tanner and J. L. Wrana, "Simultaneous Determination of Proteins Using an Element-Tagged Immunoassay Coupled with ICP-MS Detection," Journal of Analytical Atomic Spectrometry, Vol. 17, No. 8, 2002, pp. 892-896. doi:10.1039/b202306g

[31] V. I. Baranov, Z. Quinn, D. R. Bandura and S. D. Tanner, "A Sensitive and Quantitative Element-Tagged Immunoassay with ICPMS Detection," Analytical Chemistry, Vol. 74, No. 7, 2002, pp. 1629-1636. doi:10.1021/ac0110350 
[32] S. Zhang, C. Zhang, Z. Xing and X. Zhang, "Simultaneous Determination of Alpha-Fetoprotein and Free BetaHuman Chorionic Gonadotropin by Element-Tagged Immunoassay with Detection by Inductively Coupled Plasma Mass Spectrometry," Clinical chemistry, Vol. 50, No. 7, 2004, pp. 1214-1221. doi:10.1373/clinchem.2003.029850

[33] P. A. Whetstone, N. G. Butlin, T. M. Corneillie and C. F. Meares, "Element-Coded Affinity Tags for Peptides and Proteins," Bioconjugate Chemistry, Vol. 15, No. 1, 2004, pp. 3-6. doi:10.1021/bc0341501

[34] A. Tholey and D. Schaumlöffel, "Metal Labeling for Quantitative Protein and Proteome Analysis Using Inductively-Coupled Plasma Mass Spectrometry," TrAC Trends in Analytical Chemistry, Vol. 29, No. 5, 2010, pp. 399-408. doi:10.1016/j.trac.2010.01.010

[35] S. Crotti, C. Granzotto, W. R. Cairns, P. Cescon and C. Barbante, "Elemental Labeling for the Identification of Proteinaceous-Binding Media in Art Works by ICP-MS," Journal of Mass Spectrometry, Vol. 46, No. 12, 2011, pp. 1297-1303. doi:10.1002/jms.2021

[36] D. Esteban-Fernandez, C. Scheler and M. W. Linscheid,
"Absolute Protein Quantification by LC-ICP-MS Using MeCAT Peptide Labeling," Analytical and Bioanalytical Chemistry, Vol. 401, No. 12, 2011, pp. 657-666. doi:10.1007/s00216-011-5104-2

[37] D. Iwahata, K. Hirayama and H. Miyano, "A Highly Sensitive Analytical Method for Metal-Labelled Amino Acids by HPLC/ICP-MS," Journal of Analytical Atomic Spectrometry, Vol. 23, No. 8, 2008, pp. 1063-1067. doi: $10.1039 / \mathrm{b} 802862 \mathrm{a}$

[38] B. M. Peek, G. T. Ross, S. W. Edwards, G. J. Meyer, et al., "Synthesis of Redox Derivatives of Lysine and Related Peptides Containing Phenothiazine or Tris(2,2'bipyridine)ruthenium(II)," International Journal of Peptide and Protein Research, Vol. 38, No. 2, 1991, pp. 114123. doi:10.1111/j.1399-3011.1991.tb01418.x

[39] D. Iwahata, M. Tsuda, T. Aigaki and H. Miyano, "Quantitative Analysis of Branched Amino Acids in a Single Fruit Fly by LC-ICP-MS after Pre-Column Derivatization with a Metal Tag Reagent," Journal of Analytical Atomic Spectrometry, Vol. 26, No. 12, 2011, pp. 2461-2466. doi:10.1039/c1ja10212e 